\title{
Aplicativos educacionais na formação de professores
}

\author{
Anna Helena Silveira Sonego ${ }^{1}$, sonego.anna@gmail.com \\ Aline Goulart Rodrigues ${ }^{2}$, alinegrbio@gmail.com \\ Patricia Alejandra Behar ${ }^{3}$, pbehar@terra.com.br \\ ${ }^{1,3}$ Programa de Pós-Graduação em Informática na Educação (PPGIE) \\ Universidade Federal do Rio Grande do Sul (UFRGS) - Porto Alegre, RS - Brasil. \\ ${ }^{2,3}$ Programa de Pós-Graduação em Educação (PPGEDU) \\ Universidade Federal do Rio Grande do Sul (UFRGS) - Porto Alegre, RS - Brasil.
}

Resumo. Esse artigo apresenta a construção de aplicativos educacionais na formação de professores como uma possibilidade de uso dos dispositivos móveis em sala de aula para o desenvolvimento da m-learning. A cada ano aumenta a quantidade de tecnologias digitais que fazem parte do nosso cotidiano, possibilitando também o seu uso no âmbito escolar. Desse modo, percebe-se que há a necessidade de explorar, criar materiais e manipular os dispositivos móveis desde a formação inicial, para que o professor tenha subsídios teórico-práticos que, posteriormente, possam ser implementados na sua prática docente. $\mathrm{O}$ presente estudo adotou a metodologia de estudo de caso, em uma universidade no sul do brasil, onde 60 alunos de diversos cursos de licenciaturas participaram da pesquisa. Como resultados, observou-se que a utilização de dispositivos móveis, especificamente, com a construção de aplicativos, pode ser considerada uma possibilidade para inovar no processo de ensino e aprendizagem. Além disso, é capaz de potencializar a comunicação, o compartilhamento de informações, a autoria de conteúdo, criatividade e autonomia dos envolvidos nesse processo.

Palavras-chave: aplicativos educacionais; aprendizagem móvel; dispositivos móveis; formação de professores.

\section{Educational applications in teacher training}

\begin{abstract}
This article presents the construction of educational applications in teacher training as a possibility of using mobile devices in the classroom for the development of m-learning. Each year, the number of digital technologies that are part of our daily lives increases, also making it possible to use them at school. Thus, it is perceived that there is a need to explore, create materials and manipulate mobile devices since the initial training, so that the teacher has theoretical and practical subsidies that, later, can be implemented in their teaching practice. The present study adopted the case study methodology, at a university in southern Brazil, where 60 students from different undergraduate courses participated in the research. As a result, it was observed that the use of mobile devices, specifically, with the construction of applications, can be considered a possibility to innovate in the teaching and learning process. In addition, it is able to enhance communication, information sharing, content authorship, creativity and autonomy of those involved in this process.
\end{abstract}

Keywords: Educational Apps; M-learning; Mobile devices; Teacher training 


\section{Introdução}

Um grande número de pessoas de várias faixas etárias está utilizando cada vez mais os dispositivos móveis (DM) conectados à internet, em ações simples do dia-a-dia, para marcar reuniões, comunicar-se, realizar leituras de materiais ou fazer tarefas escolares. Dessa maneira, acredita-se na possibilidade de desenvolvimento de atividades que incluam estes dispositivos como mediadores nos processos educacionais, com a finalidade de fomentar a comunicação e o compartilhamento de informações de forma inovadora.

A utilização dos DM e o desenvolvimento da m-learning, aprendizagem que pode ocorrer em qualquer momento e em qualquer lugar com a utilização de DM conectados em rede de internet, tornam-se uma possibilidade emergente quando abordados nos cursos de formação de professores. Há necessidade de reformulação das práticas pedagógicas, a partir do uso dos dispositivos móveis nos ambientes educacionais, e de que estas possam tornar-se viáveis no cotidiano escolar. Estas mudanças estão associadas ao fato dos estudantes da contemporaneidade serem pertencentes às novas gerações que utilizam constantemente os DM. Nesse sentido, Twenge (2017) menciona que os jovens e adolescentes que hoje frequentam as escolas são considerados pertencentes a uma geração conectada, pelo fato de utilizarem os dispositivos a todo momento para comunicação, entretenimento, produção de conteúdo, conforme seus objetivos pessoais. Diante deste contexto, Lluna e Garcia (2017), apontam que essa geração é formada por indivíduos que nasceram em meados dos anos de 1990 a 2000. Desse modo, são sujeitos que não se imaginam viver e realizar atividades sem o uso das tecnologias digitais.

Diante deste cenário, torna-se relevante que os cursos de formação de professores oportunizem possibilidades que permitam perpassar por situações que visem explorar, criar conteúdos, manipular os DM, de modo a obter subsídios teórico-práticos que possam ser implementados posteriormente nos planejamentos de aula e ações docentes. Dessa forma, também contribui para atender a demanda da nova geração conectada. A formação de professores para a construção de aplicativos transforma o uso das tecnologias digitais em uma possibilidade para a sala de aula. Esse movimento de busca dos docentes para se atualizarem em suas práticas pode fomentar a inovação e a quebra de paradigmas ultrapassados na educação, que ainda se perpetuam. O uso de DM e aplicativos podem se tornar aliados na educação, de maneira a impulsionar diferentes formas de comunicação, de leitura, de pesquisa com vistas a fortalecer o processo de aprendizagem dos estudantes.

A partir do exposto, este trabalho tem como objetivo analisar a construção de aplicativos educacionais na formação de professores, como uma possibilidade de uso dos dispositivos móveis em sala de aula para o desenvolvimento da m-learning. Para tanto, apresenta-se a organização deste estudo em seis seções. Na segunda, é abordado o conceito de dispositivos móveis e m-learning. Na terceira, é apresentada a construção de aplicativos na formação de professores como uma possibilidade de inovação docente. $\mathrm{Na}$ quarta, aponta-se a metodologia utilizada neste estudo. Na quinta, os resultados obtidos e a discussão, e por fim, as considerações finais.

\section{Dispositivos móveis e o desenvolvimento da m-learning}

A todo o momento vivenciam-se acontecimentos que provocam mudanças nos setores econômico, social, cultural e político. Estas geram consequências e influências tanto de forma positiva quanto negativa nos seus meios e no âmbito educacional. Um exemplo a ser citado como efeito destas transições é a integração das tecnologias digitais e o uso dos dispositivos móveis, como os smartphones e tablets. Nesse sentido, acredita-se que os 
DM podem proporcionar possibilidades aos estudantes para construir e melhorar o conhecimento em qualquer tempo e espaço (Sonego et al., 2016), além de fomentar a criação de estratégias pedagógicas por meio de suas funcionalidades (Carvalho et al., 2011). Para tanto, os DM possuem potencial para desempenhar atividades escolares, através das suas possibilidades de mobilidade e a conectividade, oportunizando a busca por informações, criação e compartilhamento de conteúdos. Entretanto, destaca-se que para realizar atividades com o uso desses dispositivos nos ambientes escolares, se faz necessário que o docente tenha conhecimento sobre os DM e suas funcionalidades, para que possa planejar e implementar ações com estes aparelhos nas suas práticas docentes.

Diante deste contexto, verifica-se que os DM podem fomentar o desenvolvimento da m-learning, uma aprendizagem que cria várias "possibilidades de mediar atividades de estudo dentro e fora da sala de aula através dos dispositivos móveis, com base na definição de ensino híbrido" (Sonego, 2019, p. 52). A m-learning é desenvolvida por meio da geração de espaços de aprendizagem, que se estendem além da sala de aula tradicional, de forma a privilegiar o dinamismo e a colaboração. Além disso, esses espaços possuem como cerne as necessidades individuais. A aprendizagem por meio dos DM visa adaptar as tarefas de aprendizagem e a personalização do conteúdo dos cursos (Carvalho et al., 2011). Essa forma de aprender pode se tornar uma alternativa viável para promover situações de inovação nas práticas pedagógicas em todas as áreas do conhecimento, de maneira a possibilitar a experimentação, a utilização e a exploração das tecnologias (Behar et al., 2019).

Para promover o uso de smartphones em sala de aula, deve-se levar em consideração a necessidade de organização e planejamento das atividades escolares, de modo que o uso desta tecnologia não seja apenas um instrumento de entretenimento para os alunos, e sim, um recurso mediador do processo de ensino e aprendizagem. É importante a participação do corpo docente e da comunidade escolar nesse seguimento de difusão e desenvolvimento da m-learning (Bento; Cavalcante, 2013).

Na seção a seguir, será abordada a formação de professores como uma alternativa para os docentes que pretendem planejar e desenvolver atividades educacionais a partir do uso dos dispositivos móveis.

\section{A formação de professores e a construção de aplicativos}

A integração dos dispositivos móveis nas escolas requer, por parte dos docentes, uma reformulação da organização e dos procedimentos adotados para implementação de atividades, para que se tenha espaço para explorar, experimentar e criar com estes aparelhos. Com isso, é preciso que se estabeleça um equilíbrio na relação entre teoria e prática para potencializar o desenvolvimento desta aprendizagem. Por meio desta, os professores podem transitar por situações que ofereçam condições para planejar e implementar atividades de estudo com o uso destes aparelhos (Sonego, 2019). Nessa perspectiva, a formação de professores apresenta como objetivo a oferta de uma atualização sobre uma área ou assunto da contemporaneidade (Martins, 2008 apud Moresco, 2009).

Uma das opções existentes para formação de professores é por meio de cursos, que se tornam alternativas acessíveis para obter subsídios teórico-práticos, que são considerados necessários para esse tipo de aprendizado. Nesta perspectiva, existem possibilidades para o desenvolvimento da m-learning a partir do uso dos DM com capacidade para realização de atividades como leitura de textos, exploração das ferramentas de áudio e de vídeo, câmera fotográfica, acesso às páginas da web, entre outras funcionalidades. Além disso, potencializam o processo de ensino e aprendizagem dos estudantes dentro e fora da sala de aula (Sonego, Behar, 2019). A exploração dos V. $18 \mathrm{~N}^{\circ} 1$, julho, 2020 RENOTE

DOI: 
dispositivos permite a utilização tanto para o entretenimento quanto para ampliar as possibilidades educacionais sob orientação de um professor. A formação docente, seja inicial ou continuada, deve permitir conhecer, explorar, manusear e criar conteúdos como, por exemplo, os aplicativos educacionais.

Sendo assim, os aplicativos (App) são programas criados para os dispositivos móveis, como smartphones e tablets (Santos; Freitas, 2015). Os aplicativos educacionais têm como finalidade auxiliar no processo de ensino dentro e fora das escolas, de forma a ser uma ferramenta para diferentes áreas e que pode ser trabalhada em todas as faixas etárias. Os App podem ser encontrados em lojas online, sendo alguns pagos e outros gratuitos e apresentam grande diversidade de assuntos e possibilidades de exploração (Santos; Freitas, 2015). Além disso, para a construção de aplicativos, estão disponíveis softwares em versões gratuitas, que promovem inovação nas relações entre professor e aluno, integrando os DM no processo de ensino e aprendizagem (Behar et al., 2019).

A construção de um aplicativo educacional pode ser um desafio para os docentes, pois múltiplos aspectos devem ser considerados, bem como objetivos pedagógicos a serem atendidos. Em estudo de Krimberg et al. (2017), foram analisados os critérios considerados importantes pelos alunos de graduação para a construção de aplicativos educacionais. Esse estudo destaca, em relação aos aspectos pedagógicos, a importância da definição de objetivos educacionais, conteúdos, tipos de suporte para o aluno e meios de comunicação entre os usuários. Nos aspectos técnicos, as autoras destacam a facilidade de acesso ao download do aplicativo, a compatibilidade de diferentes tipos de dispositivos, a possibilidade de uso off-line, além de navegação e interface descomplicadas. Em relação à interação, levanta-se a importância do uso de diversos recursos visuais, sonoros e esteticamente atrativos de acordo com a faixa etária dos usuários (Krimberg et al., 2017). Todos os aspectos citados anteriormente tornam a construção de App complexa, mas que pode ser facilitada se não houver a utilização de linguagem de programação na sua confecção.

Dentre os programas disponíveis online para criação de aplicativos, evidencia-se a Fábrica de Aplicativos, pois não exige do usuário conhecimento prévio de programação e apresenta-se como poderosa ferramenta de autoria para professores e alunos, além de ser gratuito. A Fábrica de Aplicativos, presente em 120 países e com mais 500 mil App criados, foi lançada em 2012 e é uma plataforma "faça você mesmo" para construção de aplicativos, que podem ser editados e publicados por meio da Web, Android e iOS/ iPhone. Para que os App possam ser publicados e os usuários possam os instalar por meio das lojas Play Store e App Store, é necessário assinar um plano, que permite ao usuário além de poder publicar o App nas lojas online, retirar propagandas, integrar o Google Analytics e notificações push (Fábrica de Aplicativos, 2019). Nessa perspectiva, na seção a seguir será abordada a metodologia adotada neste estudo.

\section{Metodologia}

Esta pesquisa foi realizada a partir de um estudo de caso com uma abordagem qualitativa e quantitativa. Conforme Yin (2015), estudo de caso é uma forma de investigação que se refere a fenômenos, fatos e acontecimentos contemporâneos que fazem parte do nosso cotidiano. Nesse sentido, para Yin (2015): "o poder diferenciador do estudo de caso é a sua capacidade de lidar com uma ampla variedade de evidências, documentos, artefatos, entrevistas e observações, além do que pode estar disponível no estudo histórico convencional". A realização do estudo de caso abre a possibilidade, com a construção de App educacionais na formação de professores, de utilizá-los por meio de DM na sala de aula, com vistas ao desenvolvimento da m-learning. 
Os participantes dessa pesquisa são oriundos de uma disciplina eletiva ofertada para os cursos de distintas licenciaturas de uma universidade no Sul do Brasil, que visa promover os conhecimentos básicos para a inclusão das tecnologias digitais no trabalho docente. A coleta de dados foi realizada durante 2019/1 em uma turma com 30 alunos e a mesma disciplina foi replicada em 2019/2, como forma de exemplificar a prática dos estudos realizados, totalizando 60 alunos neste estudo, sendo o público composto por idades entre 18-40 anos. Para tanto, os futuros professores construíram aplicativos educacionais desenvolvendo a autoria, criatividade e autonomia por meio do uso dos dispositivos móveis. A atividade foi proposta aos futuros professores para que os mesmos planejassem e construíssem aplicativos educacionais com o tema que fosse de viável implementação nas suas ações docentes nas escolas. Sendo assim, os alunos tiveram que perpassar por várias etapas, incluindo pesquisas e leituras, para obterem subsídios para a escrita e autoria de conteúdos, como, por exemplo, a produção de vídeos, áudios, textos e outros materiais que foram incluídos nos aplicativos. O site escolhido para a construção de aplicativos foi o da Fábrica de Aplicativos (https://fabricadeaplicativos.com.br/), por não necessitar o conhecimento de linguagem de programação e ser gratuito. De tal modo, é acessível para alunos de diferentes contextos sociais e econômicos trabalharem na criação de App.

\section{Resultados e discussão}

Como resultado da produção de App durante a disciplina, os alunos criaram 20 aplicativos educacionais e trabalharam em grupos de 3 participantes. Apresentam-se abaixo quatro exemplos dentro de distintas áreas do conhecimento que ilustram os App gerados pelos professores em formação inicial.

O aplicativo Explorando a Biologia (figura 1) foi elaborado com o intuito de apresentar conteúdos de Ciências Biológicas, por meio de fotografias e textos, abordando a diversidade e a importância de conservá-la. O segundo exemplo, é de um App sobre tabela periódica (figura 2), no qual explora a história, quem foram os cientistas e suas contribuições para a Ciência, pesquisa e educação. Já o terceiro aplicativo (figura 3) foi elaborado, com o objetivo de explorar e conhecer um dos principais bairros da cidade de Porto Alegre, auxiliando os estudantes e demais indivíduos a conhecerem o que o bairro oferece como, por exemplo, escolas, postos de saúde, centro policial e outros locais de apoio à população. E por fim, o aplicativo Químaluca (figura 4), que apresenta conteúdos e atividades de ciências, tanto para educação infantil quanto para os anos iniciais do ensino fundamental. Além disso, o App é indicado para educadores, pais e estudantes por ter o intuito de disseminar o ensino de ciências de forma lúdica para todos envolvidos nesse processo. Sendo assim, destaca-se que estes aplicativos apresentam recursos, como por exemplo, a integração de textos com imagens que ajudam na explicação dos conteúdos, links com materiais de apoio, vídeos com esclarecimentos complementares e exercícios que potencializam o processo de ensino e aprendizagem. Nesse sentido, acredita-se que a construção de App pode contribuir para o desenvolvimento da autonomia dos futuros professores, a partir de novas formas de comunicação e interação durante a realização das atividades que envolvam o uso dos DM (SONEGO E BEHAR, 2015). 


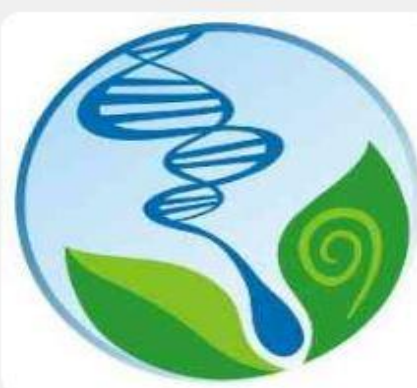

Explorando a Biologia Disciplinas

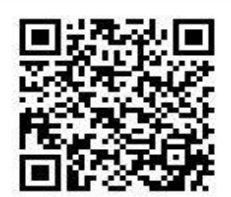

Use um leitor de QR code ou acesse https://app.vc/explorando_a_biologia pelo celular

Figura 1: Aplicativo explorando a Biologia

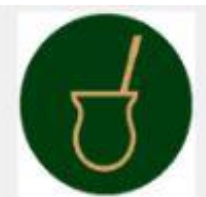

\author{
Bairro Alegre \\ Cursos
}

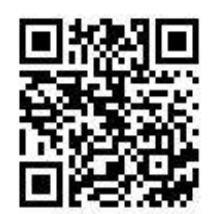

Use um leitor de QR code ou acesse https://app.vc/bairro_alegre pelo celular

Figura 3: Aplicativo Bairro Alegre

Fonte: https://galeria.fabricadeaplicativos.com.br/bairro_alegre

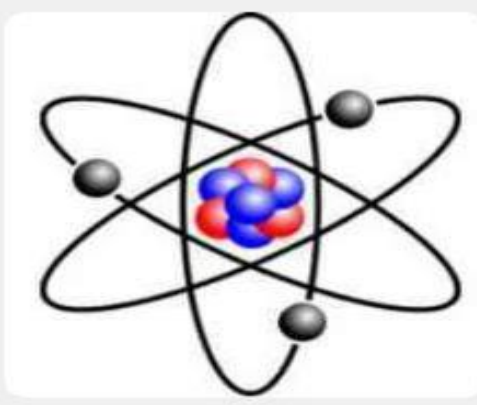

Tabela Periódica

Trabalhos Escolares

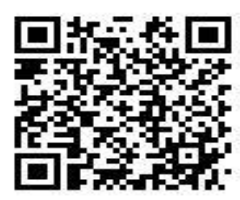

Use um leitor de QR code ou acesse https://app.vc/tabela_periodica_2090460 pelo celular

Figura 2: Aplicativo tabela periódica

Fonte:

http://galeria.fabricadeaplicativos.com.br/tabela_periodica_2090460

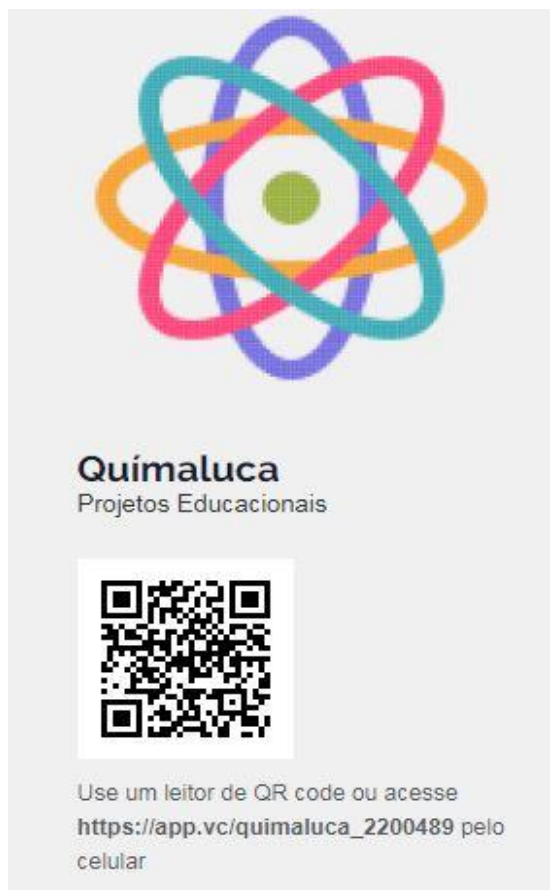

Figura 4: Aplicativo Químaluca

Fonte: https://galeria.fabricadeaplicativos.com.br/quimaluca_2200489

Os App apresentados se destacam, pois além de explorar as temáticas dentro de Biologia e Química, respectivamente, também promovem a interdisciplinaridade, pois V. $18 \mathrm{~N}^{\mathrm{o}} 1$, julho, 2020 RENOTE DOI: 
mostram aspectos do contexto histórico e também contribuições para a literatura científica. Sendo assim, Dameão et al (2017) aponta que a interdisciplinaridade na atualidade torna-se uma necessidade social no século XXI. E, se tratando da sua aplicação no ambiente escolar, esta manifesta-se como uma forma de desfragmentar o conhecimento, proporcionando um elo entre as disciplinas envolvidas, de acordo com o nível e modalidade de ensino. Portanto, a elaboração de um App, pode permitir essa integração de conteúdos de forma inovadora e criativa contribuindo tanto para formação de professores quanto para o processo de ensino e aprendizagem dos estudantes.

Nesse sentido, Silva, Silva e Silva (2015) apresentam a construção de um aplicativo para o estudo dos materiais básicos de laboratório, no qual professores de Química, que atuam em escolas públicas no estado da Paraíba, tiveram acesso e o avaliaram. O aplicativo se chama STUDYLAB, criado por meio do site Fábrica de Aplicativos. O objetivo do aplicativo foi auxiliar os alunos a se familiarizarem com os materiais básicos de laboratório. Como resultado, observaram o potencial que o aplicativo oferece para o ensino de Química, o que provocará melhorias no processo de ensino e aprendizagem nas escolas. Os autores destacam que a adoção de metodologias participativas a partir de propostas construtivistas, como a criação de Apps, pode despertar o interesse do aluno pelo estudo da Química (Silva; Silva; Silva, 2015)

Lopes e Schröder (2016) utilizaram a mesma plataforma de construção de aplicativos para a aprendizagem de ecologia por estudantes de graduação de uma universidade privada, que trabalharam em equipes para a criação de Apps. Como resultado, demonstraram que a utilização de uma plataforma livre para a construção de aplicativos para dispositivos móveis pode ser usada para melhorar o aprendizado, além de estimular competências e habilidades (Lopes; Schröder, 2016). Dessa forma, Melo e Boll (2014) evidenciam que há necessidade, com o aumento dos aplicativos educacionais, do desenvolvimento de repositórios para o mapeamento, organização, categorização e disponibilização destes aplicativos nos próprios dispositivos móveis. Além disso, os autores acreditam que o aprendizado social dessas práticas pedagógicas poderá revolucionar principalmente os desejos de permanência dos alunos nos espaços escolares, de maneira a torná-los mais interessantes para essa geração que usa os DM (Mello; Boll, 2014).

Considera-se que essa atividade de construção de um App pode oportunizar diversas contribuições na aprendizagem a partir das funcionalidades e recursos disponíveis nos smartphones e tablets, potencializando o desenvolvimento da m-learning. Além disso, destaca-se que essa atividade incentiva, nos futuros professores, o desenvolvendo da autoria, criatividade e autonomia por meio do uso dos dispositivos móveis, de modo a contribuir no processo de aprendizagem dos alunos.

\section{Conclusão}

Nesta investigação foram mostrados quatro aplicativos, desenvolvidos por docentes em formação inicial e que se tornam importantes ferramentas com potencial para dinamizar a construção do conhecimento por meio de materiais digitais. A construção de App permite que o autor desenvolva suas capacidades de leitura, escrita, criatividade e autonomia e, nesse caso, a colaboração, pois foram construídos em grupos formados por 3 participantes. Além disso, os professores em formação se basearam nos critérios pedagógicos, técnicos e interativos como referências relevantes a serem consideradas na construção de aplicativos educacionais.

$\mathrm{O}$ uso de DM no ambiente escolar é uma realidade e exige cada vez mais a formação dos docentes para que estejam preparados para mediar as informações advindas 
da Web. A aprendizagem móvel, portanto, pode oportunizar práticas e dinâmicas que visem uma maior integração entre os alunos e os docentes. Para tanto, é relevante que os professores perpassem durante sua formação, seja inicial ou continuada, por situações que permitam explorar os DM, para que tenham subsídios teórico-práticos para realizarem o planejamento e a implementação de atividades escolares usando essa tecnologia, como por exemplo, a criação de aplicativos educacionais.

Portanto, a construção de Apps educacionais por docentes torna-se uma necessidade no cotidiano escolar, que exige cada vez mais atualização para a utilização desses recursos, de modo que possam ajudar seus alunos a selecionarem as informações advindas da rede. $\mathrm{O}$ uso de plataformas gratuitas e que não usam a linguagem de programação pode facilitar o acesso às possibilidades de criação por parte dos professores, de modo a promover a inovação das práticas pedagógicas.

\section{Referências}

BEHAR, P. A. Recomendação Pedagógica em Educação a Distância. Porto Alegre: Penso, 2019. 208 p.

BENTO, M. C. M.; CAVALCANTE, R. S. Tecnologias móveis em educação: o uso do celular na sala de aula. ECCOM, Lorena, SP, v.4, n.7, p.113-120, 2013.

CARVAlhO, A. S.; BASTOS, H. P. P.; LEITE, M. L. F. T. T; DA COSTA, R. G. Educação e Tecnologia: um percurso interinstitucional. Campos dos Goytacazes: Essentia Editora, 2011. 272p.

DAMEÃO, A. P.; ROSA, P. R. S.; ERROBIDART, N. C. G. Um método para o trabalho interdisciplinar na escola. Revista Fórum Identidades. Itabaiana-SE,v. 25, p. 37-54, set.-dez. 2017.2 Disponível em: < https://seer.ufs.br/index.php/forumidentidades/article/view/8341> Acesso em: 13 jul. 2020.

FÁBRICA DE APLICATIVOS. Sobre a Fábrica de Aplicativos, 2019. Disponível em: $<$ https://suporte.fabricadeaplicativos.com.br/hc/pt-br/articles/360000530606-Sobrea-F\%C3\%A1brica-de-Aplicativos>. Acesso em: 17 out 2019.

KRIMBERG, L.; SONEGO, A. H. S.; RIBEIRO, A. C. R.; BEHAR, P.A. Construção de aplicativos educacionais na formação de professores: critérios pedagógicos, técnicos e interativos. In: XXII Conferência Internacional de Informática na Educação, volume 13, 2017. Anais TISE. Santiago do Chile, p.144-149. Disponível em: <http://www.tise.cl/2017/img/ActasTISE2017.pdf> Acesso em: 18 out. 2019.

LOPES, L. A.; SCHRÖDER, N. T. A elaboração de aplicativos para dispositivos móveis como prática educativa no ensino de Ecologia. Revista Tecnologias na Educação, v.17, n. 8, p. 1-11, 2016.

LLUNA, S.; GARCIA, J. P. Introducción. In: LLUNA, S.; PEDREIRA, J. (ed.). Los nativos digitales no existen: Cómo educar a tus hijos para un mundo digital. Barcelona: Deusto, 2017. p. 27-31. 
MARTINS, A. R, 2009. Não Basta (só) tapar buracos: nas redes públicas, faltam programas preocupados, de fato, com a continuidade da capacitação docente. Nova Escola, São Paulo, Ano XXIII, No 216, p. 54 - 57, outubro, 2008. In: MORESCO, S. F. S. Tomada de consciência sobre o trabalho com projetos a partir da utilização de um objeto de aprendizagem integrado a um ambiente virtual de aprendizagem. Porto Alegre: UFRGS, 2009. 250p. Tese de Doutorado. Disponível em: 〈http://www.lume.ufrgs.br/handle/10183/21390>. Acesso em: 21 mar. 2019.

MELO, R. S.; BOLL, C. I. Cultura Digital e Educação: desafios contemporâneos para a aprendizagem escolar em tempos de dispositivos móveis. RENOTE-Revista Novas Tecnologias na Educação, v. 12, n. 1, p.1-11, 2014.

SANTOS, F. M. V.; FREITAS, S. F. Avaliação da usabilidade de ícones de aplicativo móvel utilizado como apoio educacional para crianças na idade pré-escolar. Ação Ergonômica, [S.1.], v. 10, n. 2, p.123-132, 2015.

SILVA, P. F.; SILVA, T. P.; SILVA, G. N. StudyLab: Construção e Avaliação de um aplicativo para auxiliar o Ensino de Química por professores da Educação Básica. Revista Tecnologias na Educação, v. 13, n. 7, p. 1-12, 2015.

SONEGO, A. H. S., MACHADO, L. R.; TORREZZAN, C. A. W.; BEHAR, P. A. Mobile Learning: Pedagogical Strategies for Using Applications in the Classroom. 12th International Conference on Mobile Learning. Vilamoura, Algarve, Portugal, 2016, p.28-34.

SONEGO, A. H. S. ARQPED-MOBILE: Uma arquitetura pedagógica com foco na aprendizagem móvel. Porto Alegre: UFRGS, 2019. 241p. Tese de Doutorado Disponível em: <https://lume.ufrgs.br/handle/10183/197443>. Acesso em: $10 \mathrm{mar}$ 2020.

SONEGO, A. H. S.; BEHAR, P. A. M-learning: o uso de dispositivos móveis por uma geração conectada. Revista Educação (Porto Alegre), v. 42, n. 3, p. 514-524, set.dez. 2019. Disponível em:

<http://revistaseletronicas.pucrs.br/ojs/index.php/faced/article/view/32203>. Acesso em: 15 mar 2020.

SONEGO, A. H. S.; BEHAR, P. A. M-learning: reflexões e perspectivas com o uso de aplicativos educacionais. In: $\mathbf{X X}$ Congresso Internacional de Informática Educativa, v. 11, 2015. Anais TISE. Santiago do Chile, p. 521-526. Disponível em: <http://www.tise.cl/volumen11/TISE2015/521-526.pdf>

TWENGE, J. M. iGen: why today's super-connected kids are growing up less rebellious, more tolerant, less happy and completely unprepared for adulthood. New York: Atria Books, 2017.

YIN, R. K. Estudo de Caso: planejamento e métodos. Tradução: Cristhian Matheus Herrera. $5^{\text {a }}$ ed. Porto Alegre: Bookman, 2015. 